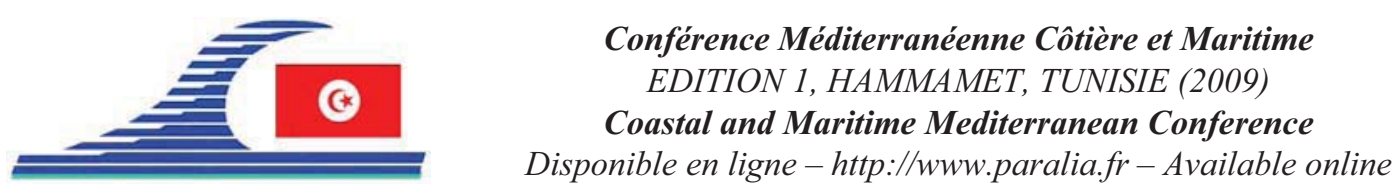

\title{
Efficacité d'un rechargement de plage en milieu fortement anthropisé : le cas de la plage du Ceinturon (Hyères, Var)
}

\author{
Romain CAPANNI ${ }^{1}$, François SABATIER ${ }^{1}$
}

1. Aix-Marseille Université, CEREGE UMR 6635 Europôle Méditerranéen de l'Arbois BP 80 - Cedex 04, 13545 Aix-en-Provence, France.

capanni@cerege.fr; sabatier@cerege.fr

\section{Résumé :}

Les plages sableuses représentent près de $40 \%$ des littoraux du monde et près de $75 \%$ d'entre elles sont sujettes à l'érosion (BIRD, 1996). Dans ce contexte, de nombreuses techniques de protection ont été mise en place (enrochements, épis, brise-lames...) dans le but de réduire l'énergie des vagues approchant le littoral ou de piéger le sédiment en transit. Cependant, ces protections ne sont que partielles et parfois les ouvrages exacerbent les problèmes qu'ils étaient censé résoudre (SABATIER et al. 2009). La plage du Ceinturon (Var, méditerranée), connaît plusieurs types de méthodes de «protection côtière » : des enrochements (épis et digues) depuis les années 1970 et des rechargements artificiels ponctuels depuis les années 1990. Dans cette présentation nous montrons les limites de ces deux méthodes. Les enrochements induisent une érosion en aval dérive caractéristique (SAMAT, 2007), mais cette dernière, combattue par les rechargements artificiels, n'est pas stoppée en raison de volumes de dépôts insuffisants.

\section{Mots-clés :}

Littoral - Erosion - Epis - Digue - Rechargement - Hard engineering - Soft engineering

\section{Introduction}

Face aux nombreux inconvénients engendrés par les enrochements, le rechargement artificiel de plage est devenu peu à peu une alternative pour les littoraux dont le rivage recule (HAMM et al., 2002). Cette présentation analyse l'efficacité de ces deux types de techniques d'ingénierie sur la plage du Ceinturon (Hyères, méditerranée).

\section{Présentation du site}

La plage du Ceinturon à Hyères les Palmiers est située en rade de Hyères (Var, Méditerranée), dont le littoral s'étend sur près de $25 \mathrm{~km}$ (figure 1). La branche orientale $\mathrm{du}$ tombolo est constituée de plages à sédiments mixtes (sables, graviers et galets) au nord qui s'affinent en allant vers le sud en accord avec la dérive littorale dominante dirigée dans cette direction. 


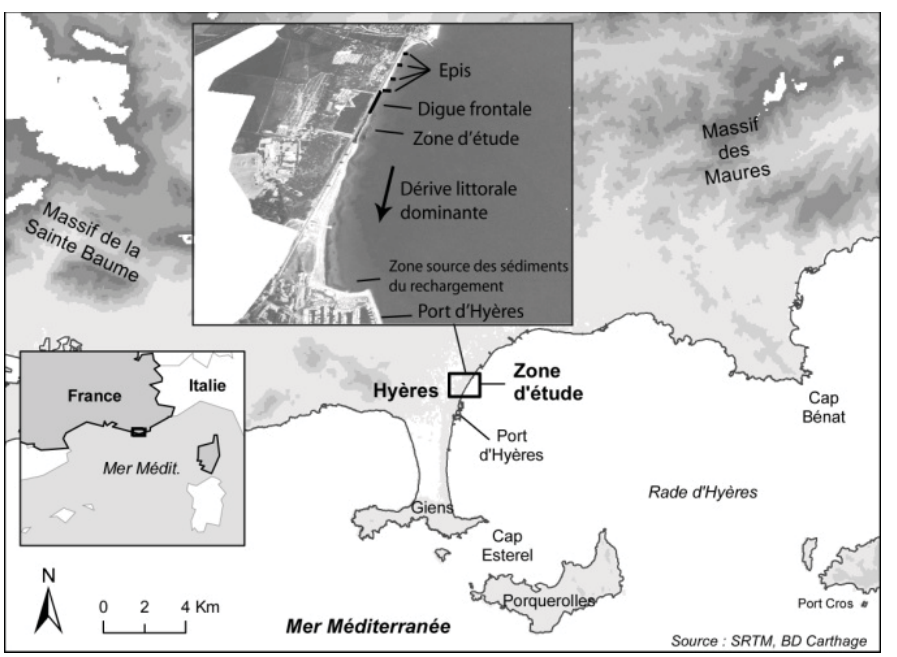

Figure 1. Carte de localisation.

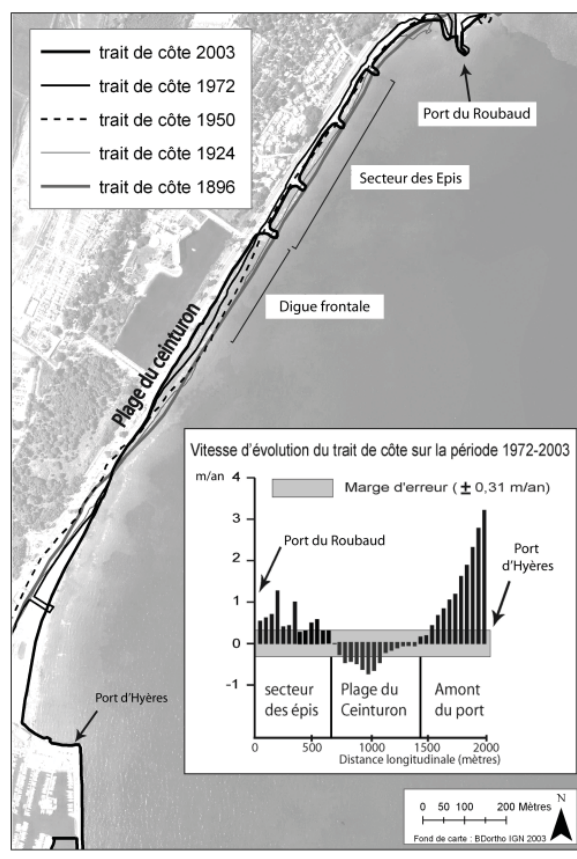

Figure 2. Evolution et vitesses d'évolution du trait de côte entre 1972 et 2003.

L'accélération de l'anthropisation à partir de la fin des années soixante (extension des ports, constructions d'épis, calibrage des cours d'eau...) a modifié de façon importante le fonctionnement sédimentaire de ce littoral qui est aujourd'hui contrôlé par les digues portuaires qui le segmentent. La plage du Ceinturon est située en aval dérive d'une digue frontale longue de $200 \mathrm{~m}$ et d'une batterie de 4 épis longs de $50 \mathrm{~m}$ et espacés de $150 \mathrm{~m}$ construits à la fin des années 1970. Elle est également située en amont dérive du port d'Hyères édifié dans les années 1950 et dont la digue nord, longue de $300 \mathrm{~m}$, bloque la quasi-totalité du transit sédimentaire. Longue de $300 \mathrm{~m}$ et large de $20 \mathrm{~m}$, cette plage est naturellement constituée de sables fins.

\section{Méthodologie}

L'étude de l'évolution du trait de côte entre 1896-1924-1950-1972 et 2003 s'inspire de la méthodologie classique de l'analyse diachronique de l'évolution des littoraux sous S.I.G. et les variations du rivage sont analysées à l'aide du module Digital Shoreline Analysis System de Arcgis (THIELER et al., 2005). L'analyse de l'évolution topographique à court terme de la plage du Ceinturon par modèle numérique de terrain (MNT) repose sur des relevés réalisés au DGPS en mai, juillet, septembre, novembre et décembre 2008. La marge d'erreur est fixée à $+/-10 \mathrm{~cm}$, celle-ci étant largement supérieure à l'erreur réelle mesurée par comparaison de différents relevés sur des points fixes (dalles en béton). 


\section{Résultats et discussion}

Entre 1896 et 1972, le secteur en aval du port du Roubaud est marqué par une importante érosion (-0.4 m/an environ) (figure 2). Par contre, à partir de 1972, la construction de 4 épis entre lesquels des rechargements en galets ont été réalisé, a permis de stopper le recul du rivage et d'inverser la tendance ( $+0.52 \mathrm{~m} / \mathrm{an}$ en moyenne). En revanche, en aval dérive, au niveau de la plage du Ceinturon, l'érosion se poursuit $(-0.35 \mathrm{~m} / \mathrm{an}$ environ). La plage du Ceinturon est également située en amont de la zone d'influence de la digue nord du port d'Hyères qui permet une importante accumulation depuis les années 1950 ; le trait de côte avance d'environ 1.35 m/an entre 1972 et 2003. Ce secteur constitue une zone de stockage sédimentaire d'où sont prélevés des sables utilisés pour le rechargement artificiel de la plage du Ceinturon.

Les relevés topographiques en début d'été (mai-juillet 2008), indiquent d'abord un arasement anthropique $\left(450 \mathrm{~m}^{3}\right)$ du talus de protection qui servait à limiter les inondations marines puis un dépôt artificiel de rechargement en sables $\left(250 \mathrm{~m}^{3}\right)$. Durant l'été (juillet-septembre 2008), le différentiel topographique indique un bilan sédimentaire négatif de $-40 \mathrm{~m}^{3}$ (accrétion : $20 \mathrm{~m}^{3}$; érosion $60 \mathrm{~m}^{3}$ ) en relation avec l'érosion des dépôts artificiels (figure $3 b$ ). La période estivale est donc marquée par un rééquilibrage du profil de plage, les sédiments déposés sur la berme lors du rechargement sont exportés vers la plage sous-marine. En revanche, lors des premières tempêtes automnales (septembre-novembre 2008) (figure 3c), la plage affiche de fortes pertes sédimentaires difficiles à quantifier car le talus de protection a été reconstruit par la municipalité en mélangeant des dépôts produits par les vagues et du remblai anthropique. La plage, non affectée par le recalibrage du talus de protection, montre une érosion dominante de $170 \mathrm{~m}^{3}$ tandis que la morphologie abrupte du talus témoigne d'un début de sapement par la houle. L'érosion aval dérive est aussi caractéristique pendant cette période. Durant les tempêtes hivernales, (novembre-décembre 2008), l'érosion est plus forte et induit un bilan net déficitaire de $480 \mathrm{~m}^{3}$ environ (figure 3d). Le talus de «protection » est détruit et des laisses de tempêtes envahissent la route située en arrière de la plage. Tout comme entre septembre et novembre l'érosion est particulièrement marquée en aval immédiat des ouvrages en enrochements (figures $3 c$ et $3 \mathrm{~d}$ ).

\section{Conclusion}

La situation de la plage du Ceinturon, en aval dérive d'ouvrages, est problématique pour les gestionnaires depuis la mise en place des enrochements. Ces équipements sont responsables d'une importante érosion laquelle est actuellement combattue à l'aide de rechargements aux volumes limités. Cependant, le rechargement en sédiments fin n'est qu'une solution temporaire qui ne permet de recréer une plage indispensable à l'activité balnéaire estivale que très ponctuellement dans le temps. En effet, la dynamique érosive engendrée par les houles de beau temps prend rapidement l'ascendant provoquant une 
Meilleures pratiques environnementales en ingénierie côtière et maritime

disparition des sédiments rechargés avant l'automne. Les tempêtes automnales et hivernales érodent ensuite fortement la plage.

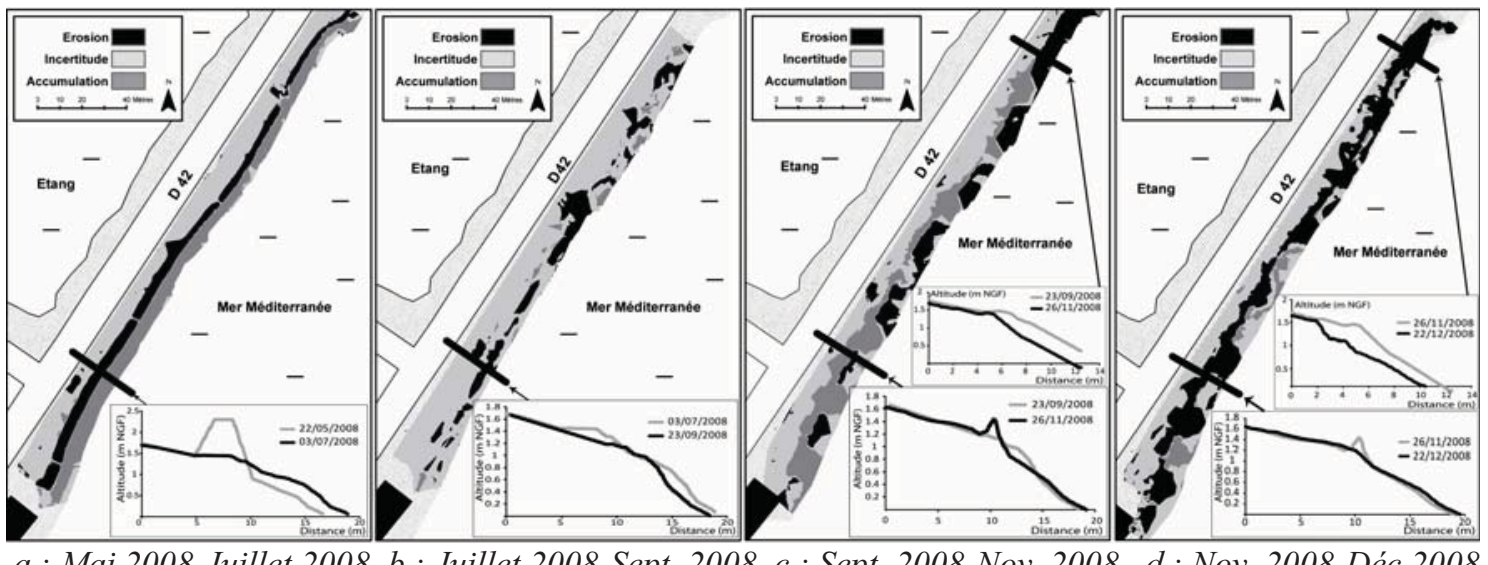

a : Mai 2008-Juillet 2008 b : Juillet 2008-Sept. 2008 c : Sept. 2008-Nov. 2008 d: Nov. 2008-Déc.2008

Figure 3. Evolution topographique de la plage du Ceinturon.

\section{Références bibliographiques}

BIRD E.C.F. (1996). Beach management. Chichester : Wiley and sons.

HAMM L., CAPOBIANCO M., DETTE H.H., LECHUGA A., SPANHOFF R., STIVE M. J. F. (2002). A summary of European experience with shore nourishment. Coastal Engineering, 47, 2, pp 237-264.

SABATIER F., SAMAT O., ULLMANN A., SUANEZ S. (2009). Conecting largescale coastal behaviour with coastal management of the Rhône delta. Geomorphology, 107, pp 79-89.

SAMAT O. (2007). Efficacité et impact des ouvrages en enrochements sur les plages microtidales. Le cas du Languedoc et du delta du Rhône. Thèse Université de Provence, $371 \mathrm{p}$.

THIELER E.R., HIMMELSTOSS E.A., ZICHICHI J.L., MILLER T.L. (2005). Digital Shoreline Analysis System (DSAS) version 3.0; An ArcGISC extension for calculating shoreline change. U.S. Geological Survey Open-File Report, 1304. 